\title{
Qualité radiologique des eaux filtrées du Rhône aval en vue de la production d'eau destinée à la consommation humaine
}

\section{Mise en perspective historique et régionale}

\author{
F. Eyrolle-Boyer ${ }^{1 \text { a }}$, Ph. Renaud ${ }^{1}$, D. Claval ${ }^{1}$, D. Tournieux ${ }^{2}$, F. Le Dore ${ }^{3}$, J.-F. Blanchet ${ }^{3}$, J. Loyen $^{2}$, \\ Ch. Antonelli ${ }^{1}$, C. Cossonnet ${ }^{4}$ et X. Cagnat ${ }^{4}$ \\ 1 IRSN, PRP-ENV, SESURE/LERCM, BP 3, 13115 Saint Paul Lez Durance, France. \\ 2 IRSN, PRP-ENV, SESURE/LS3E, LTE, 31 rue de l'Écluse, BP 35, 78116 Le Vésinet, France. \\ 3 BRL Direction Générale, 1105 avenue Pierre Mendès France, BP 94001, 30001 Nîmes Cedex 5, France. \\ 4 IRSN, PRP-ENV, STEME/LMRE, Bât. 501, Le Bois des Rames, 91400 Orsay, France.
}

Reçu le 8 avril 2013 - Accepté le 19 mai 2014

Résumé - Le code de la santé publique et notamment l'arrêté du 11 janvier 2007, relatif aux limites et références de qualité des eaux brutes et des eaux destinées à la consommation humaine, fixe quatre indicateurs de la qualité radiologique des eaux du robinet (l'activité $\alpha$ globale, l'activité $\beta$ globale, l'activité du tritium et la dose totale indicative), ainsi que des valeurs guides et des références de qualité. Les chroniques issues de la surveillance des eaux filtrées du Rhône aval montrent que, si la radioactivité d'origine naturelle demeure bien évidemment constante au cours du temps, les niveaux de contamination radioactive d'origine artificielle ont fortement diminué à partir du début des années 90 , de 10 à 100 fois suivant les radionucléides. Les données soulignent également qu'aucune des limites d'activités $\alpha$ globale, $\beta$ globale et en tritium n'a été dépassée dans l'eau filtrée du Rhône aval au cours de l'ère industrielle nucléaire. Les doses totales indicatives (DTI) calculées à partir des prélèvements d'eau filtrée du Rhône aval (canal Philippe Lamour - Réseau hydraulique régional propriété de la région Languedoc Roussillon géré par BRL), de l'Orb et de l'Hérault effectués en 2011 sont très inférieures à la valeur de référence de $100 \mu \mathrm{Sv} / \mathrm{an}$. La contribution à la DTI des radionucléides artificiels détectés dans ces hydrosystèmes est en outre négligeable $(<0,01 \%)$.

\begin{abstract}
Radiological quality Assessment of Rhône River filtered waters at its lower course in the framework of water production for human consumption with historical and regional perspectives. The public health code, including the Ministerial Decree of 11 June 2007, set four indicators to evaluate the radiological quality of drinking waters ( $\alpha$ gross activity, $\beta$ gross activity, tritium activity and total indicative dose), as well as guide values and quality references. All of the data from the monitoring show that while the radioactivity of the natural origin course remained constant over time, the levels of radioactive contamination of the Rhône from artificial sources declined sharply from the early 90s, i.e. 10 to 100 times following radionuclides. The data also emphasise that no threshold activities for $\alpha$ global, $\beta$ global and tritium was exceeded in filtered water of the Rhône. The total indicative dose (TID) remained below $100 \mu \mathrm{Sv} /$ year. TID quantification from water samplings in 2011 in the downstream part of the Rhône River (BRL channel), Orb river and Hérault river are all far below the quality references. The contribution to the TID of artificial radionuclides detected in these water systems was shown to be negligible $(<0.01 \%)$.
\end{abstract}

Keywords: Radiological quality / drinking waters / Total Indicative Dose (TID) / gross activity / Rhône River / Orb River / Hérault River

\section{Introduction}

En France, l'exposition aux rayonnements ionisants d'origine naturelle est en moyenne de 2,4 mSv/an dont environ seulement $0,2 \mathrm{mSv} / \mathrm{an}$ sont dus à l'ingestion d'aliments et d'eau (IRSN, 2006). Par ailleurs, les modalités de contrôle de

\footnotetext{
a frederique.eyrolle-boyer@irsn.fr
}

la qualité radiologique des eaux destinées à la consommation humaine sont fixées par l'arrêté du 11 janvier 2007, relatif aux limites et références de qualité des eaux brutes et des eaux destinées à la consommation humaine (CSP, 2007). Cet arrêté fixe les indicateurs radiologiques : l'activité $\alpha$ globale, l'activité $\beta$ globale, l'activité du tritium et la dose totale indicative (DTI). La DTI est la dose efficace engagée résultant pour un adulte de l'incorporation de tous les radionucléides naturels 
Tableau 1. Indicateurs radiologiques règlementaires permettant d'évaluer la qualité radiologique des eaux destinées à la consommation humaine.

Radiological indicators used according to regulatory requirements in order to estimate the radiological quality of waters for human consumption.

\begin{tabular}{ccc}
\hline Indicateurs & Valeur guide & $\begin{array}{c}\text { Référence } \\
\text { de qualité }\end{array}$ \\
\hline Activité alpha globale & $0,1 \mathrm{~Bq} / \mathrm{L}$ & \\
\hline Activité bêta globale résiduelle & $1 \mathrm{~Bq} / \mathrm{L}$ & \\
Activité du tritium & & $100 \mathrm{~Bq} / \mathrm{L}$ \\
\hline Dose Totale Indicative (DTI) & & $0,1 \mathrm{mSv} / \mathrm{an}$ \\
\hline
\end{tabular}

et artificiels détectés dans de l'eau de distribution consommée à raison de $2 \mathrm{~L} / \mathrm{j}$ sur une période d'un an. Elle exprime l'exposition aux rayonnements ionisants et permet de rendre compte du contrôle sanitaire de la qualité radiologique des eaux. Si l'eau distribuée ne dépasse pas $0,1 \mathrm{~Bq} / \mathrm{L}$ en activité $\alpha$ globale, $1 \mathrm{~Bq} / \mathrm{L}$ en activité $\beta$ globale résiduelle et $100 \mathrm{~Bq} / \mathrm{L}$ de tritium, la DTI ne dépasse pas la valeur de référence de $0,1 \mathrm{mSv} / \mathrm{an}$. Si les activités dépassent ces limites, la DTI est susceptible de dépasser cette valeur de référence et des mesures complémentaires doivent être réalisées, en particulier afin d'identifier l'origine de la contamination.

La vallée du Rhône a été le siège du développement de l'industrie nucléaire dès le milieu du siècle dernier. Durant des décennies l'ensemble du cycle du combustible nucléaire y était représenté, faisant du Rhône l'un des fleuves des plus nucléarisés au monde. Les eaux du Rhône constituent une ressource stratégique pour l'irrigation et l'alimentation en eau potable des territoires du sud-est de la France, en particulier en région Languedoc Roussillon via son Réseau hydraulique régional propriété de la région dont le canal Philippe Lamour géré par BRL. L'exploitation des données acquises en 2011 sur les eaux filtrées du canal ainsi que celles enregistrées par les stations de surveillance de l'IRSN depuis plusieurs décennies permet d'évaluer la qualité radiologique de l'eau. Une mise en perspective avec les ressources en eau des fleuves côtiers Orb et Hérault est également présentée.

\section{Matériel et Méthode}

\subsection{Réglementation}

Le code de la santé publique et les textes pris pour application, transposant la directive européenne 98/83/CE, fixent les modalités du contrôle sanitaire des eaux destinées à la consommation humaine. L'arrêté ministériel du 11 juin 2007 fixe quatre indicateurs de la qualité radiologique des eaux du robinet ainsi que des valeurs guides et des références de qualité (Loyen et Thomassin, 2009) (Tab. 1).

- L'activité alpha globale est représentative de l'activité de l'ensemble des radionucléides émetteurs de rayonnements alpha contenus dans l'eau. Une valeur guide de $0,1 \mathrm{~Bq} / \mathrm{L}$ est associée à cet indicateur.
- L'activité bêta globale résiduelle est représentative de l'activité de l'ensemble des radionucléides émetteurs de rayonnements bêta contenus dans l'eau, à l'exception de celle du potassium 40. Activité bêta globale résiduelle $=$ Activité bêta globale mesurée $-27,9 \times 10^{-3} \times[\mathrm{K}]$, avec $[\mathrm{K}]$ : concentration en potassium total (en $\mathrm{mg} / \mathrm{L}$ ).

Une valeur guide de $1 \mathrm{~Bq} / \mathrm{L}$ est associée à cet indicateur.

- Le tritium est un indicateur de radioactivité issue d'activités anthropiques. La valeur de référence de qualité associée à cet indicateur est de $100 \mathrm{~Bq} / \mathrm{L}$.

- La dose totale indicative (DTI) représente la dose efficace résultant de l'incorporation des radionucléides présents dans l'eau durant une année de consommation, à l'exclusion de ceux à vie courte résultant de la désintégration du radon et figurant en annexe de l'arrêté du 12 mai 2004. Elle est obtenue par le calcul en considérant une consommation quotidienne d'eau de 2 litres. La valeur de référence de qualité associée à cet indicateur est de $0,1 \mathrm{mSv} / \mathrm{an}$.

Si l'eau distribuée ne dépasse pas $0,1 \mathrm{~Bq} / \mathrm{L}$ en activité $\alpha$ globale, $1 \mathrm{~Bq} / \mathrm{L}$ en activité $\beta$ globale résiduelle et $100 \mathrm{~Bq} / \mathrm{L}$ de tritium, la DTI ne dépasse pas la valeur de référence de $0,1 \mathrm{mSv} / \mathrm{an}$. Si les activités dépassent ces limites, la DTI est susceptible de dépasser cette valeur de référence et des mesures complémentaires doivent être réalisées pour caractériser les activités des radionucléides en cause et évaluer plus précisément la DTI. Les valeurs d'activités $\alpha$ et $\beta$ globales et de tritium précédentes constituent donc des seuils d'investigation (valeurs guide), et non pas des limites de potabilité.

\subsection{Calcul des DTI}

La DTI est évaluée par la relation suivante :

$$
\mathrm{DTI}=\Sigma \mathrm{A} \times \mathrm{DPUI} \times \mathrm{q}
$$

avec : A, l'activité du radionucléide mesurée dans l'eau (en $\mathrm{Bq} / \mathrm{L}$ ), DPUI la Dose Par Unité d'Incorporation ou facteur de dose (en $\mathrm{Sv} / \mathrm{Bq}$ ) et q le volume d'eau potable consommé par an, soit $730 \mathrm{~L}$. Les valeurs des DPUI en cas d'ingestion par un adulte du public utilisées dans cette étude sont extraites de la publication 72 (ICRP, 1995).

\subsection{Prélèvements et analyses}

La radioactivité de l'eau du Rhône fait l'objet d'une surveillance par l'IRSN depuis les années 60. L'une des stations de prélèvement est à Vallabrègues, à une vingtaine de kilomètres en amont de Fourques où se situe la prise d'eau du canal (Fig. 1). Plus à l'aval, une station observatoire du Rhône en Arles (SORA), permet de mesurer des radionucléides artificiels présents à l'état d'ultra traces dans l'eau filtrée du Rhône grâce à la réduction par évaporation de grands volumes d'eau filtrée (Antonelli et al., 2012; Eyrolle et al., 2012). Ce traitement permet d'obtenir des facteurs de concentrations de 4000 à 9000 et d'abaisser ainsi les limites de détection. 


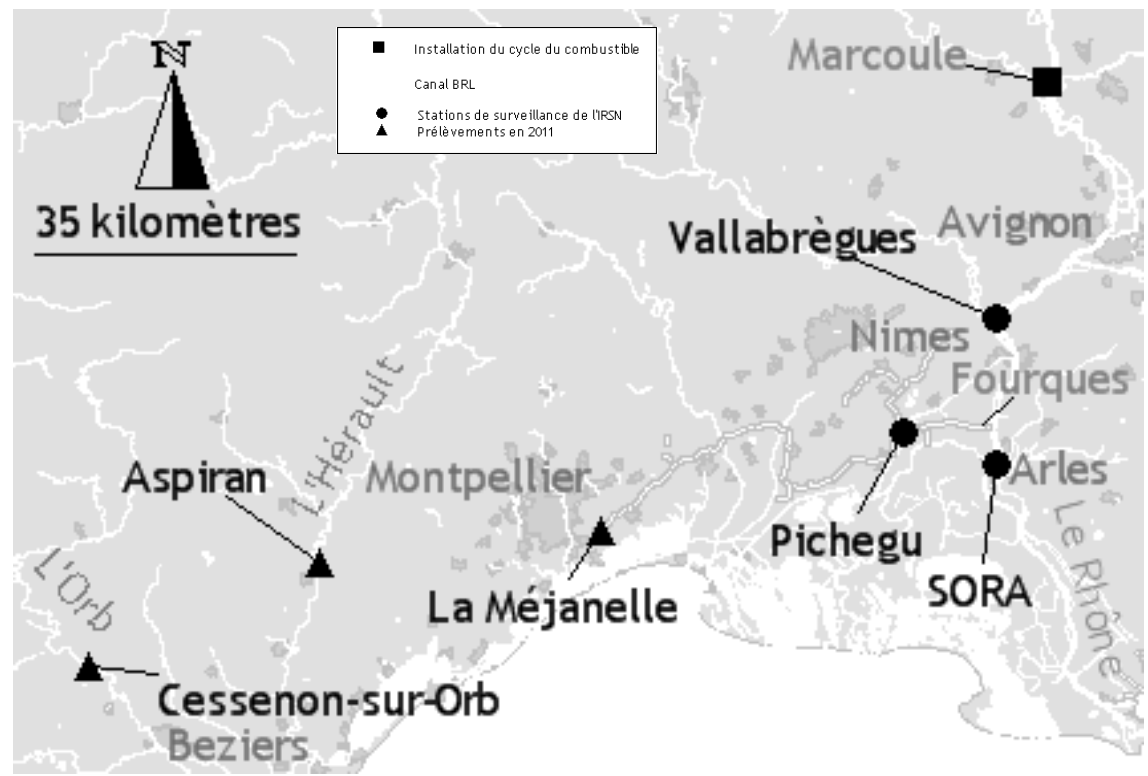

Fig. 1. Localisation des points de suivi et de prélèvements d'eau filtrée dans la partie terminale du fleuve Rhône (Rhône aval), le canal BRL et les fleuves côtiers Orb et Hérault.

Location of sampling points at the lower course of the Rhone River, the Philippe Lamour channel and the Orb and Hérault coastal rivers.

Tableau 2. Caractéristiques des échantillons étudiés et analyses effectuées.

Sample characteristics and analyses.

\begin{tabular}{|c|c|c|c|c|}
\hline Fleuve/Localisation & $\begin{array}{c}\text { Coordonnées WGS } 84 \\
\text { Latitude / Longitude }\end{array}$ & $\begin{array}{l}\text { Date de } \\
\text { prélèvements ou } \\
\text { période de suivi }\end{array}$ & Qualité & Analyses \\
\hline $\begin{array}{l}\text { Rhône aval/Arles } \\
\text { (SORA) }\end{array}$ & $43,678690^{\circ} / 4,621150^{\circ}$ & depuis 2002 & Eau filtrée & $\begin{array}{l}\text { Spectrométrie gamma, spectrométrie alpha } \\
\text { (isotopes } \mathrm{Pu}, \mathrm{Am}, \mathrm{Cm}),{ }^{90} \mathrm{Sr} \text {, tritium }\left({ }^{3} \mathrm{H}\right)\end{array}$ \\
\hline $\begin{array}{c}\text { Rhône } \\
\text { aval/Vallabrègues }\end{array}$ & $43,856285^{\circ} / 4,620094^{\circ}$ & depuis 1978 & Eau filtrée & $\begin{array}{c}\text { Alpha global, bêta global, spectro gamma, }{ }^{90} \mathrm{Sr} \text {, } \\
\text { tritium }\left({ }^{3} \mathrm{H}\right)\end{array}$ \\
\hline $\begin{array}{l}\text { Rhône aval/Canal } \\
\text { BRL (Pichegu) }\end{array}$ & $43,716580^{\circ} / 4,463805^{\circ}$ & 1985-1997 & Eau filtrée & $\begin{array}{l}\text { Alpha global, bêta global, spectrométrie gamme, }{ }^{90} \mathrm{Sr} \text {, } \\
\text { tritium }\left({ }^{3} \mathrm{H}\right) \text {, radium }\left({ }^{226} \mathrm{Ra}\right), \mathrm{U} \text { pondéral, potassium }\end{array}$ \\
\hline $\begin{array}{l}\text { Rhône aval/Canal } \\
\text { BRL (La Méjanelle) }\end{array}$ & $43,597583^{\circ} / 3,952836^{\circ}$ & 08/12/2011 & Eau filtrée & $\begin{array}{c}\text { Alpha global, bêta global, bêta global résiduel, DTI, } \\
\text { spectrométrie alpha (isotopes Pu et Am), }{ }^{90} \mathrm{Sr},{ }^{210} \mathrm{Po} \text {, } \\
\text { tritium }\left({ }^{3} \mathrm{H}\right) \text {, spectrométrie gamma, ICPMS } \\
\text { (isotopes U et Th), potassium }\end{array}$ \\
\hline Hérault (Aspiran) & $43,557509^{\circ} / 3,482397^{\circ}$ & 04/10/2011 & Eau filtrée & $\begin{array}{c}\text { Alpha global, bêta global, bêta global résiduel, DTI, } \\
\text { spectrométrie alpha (isotopes Pu et Am), }{ }^{90} \mathrm{Sr},{ }^{210} \mathrm{Po} \text {, } \\
\text { tritium }\left({ }^{3} \mathrm{H}\right) \text {, spectrométrie gamma, ICPMS } \\
\text { (isotopes U et Th), potassium }\end{array}$ \\
\hline $\begin{array}{l}\text { Orb (Cessenon-sur- } \\
\text { Orb) }\end{array}$ & $43,436997^{\circ} / 3,090134^{\circ}$ & $12 / 10 / 2011$ & Eau filtrée & $\begin{array}{l}\text { Alpha global, bêta global, bêta global résiduel, DTI, } \\
\text { spectrométrie alpha (isotopes Pu et Am), }{ }^{90} \mathrm{Sr} \text {, } \\
{ }^{210} \mathrm{Po} \text {, tritium }\left({ }^{3} \mathrm{H}\right) \text {, spectrométrie gamma, ICPMS } \\
\text { (isotopes U et Th), potassium }\end{array}$ \\
\hline
\end{tabular}

Le canal Philippe Lamour, au niveau de la station de pompage A. Dumont (Pichegu), a fait l'objet de 1982 à 1997, d'une surveillance similaire à celle effectuée à Vallabrègues. En 2011, des prélèvements ponctuels d'eau filtrée ont été réalisés au sein du canal au niveau de la station de pompage de la Méjanelle ainsi que sur les fleuves Orb, à proximité de Cessenon-sur-Orb, et Hérault aux alentours d'Aspiran. Le tableau 2 synthétise la localisation et les caractéristiques des échantillons étudiés ainsi que les analyses effectuées. Les méthodes de traitement et de conditionnement des échantillons pour analyses sont consultables dans Eyrolle et Renaud (2012).

\section{Résultats et discussion}

\subsection{Niveaux de radioactivité dans les eaux filtrées du Rhône aval}

\section{Les données du réseau de surveillance de l'IRSN}

La figure 2 présente les activités des principaux radionucléides artificiels présents dans l'eau filtrée du Rhône mesurés à Vallabrègues et à Arles (SORA). 

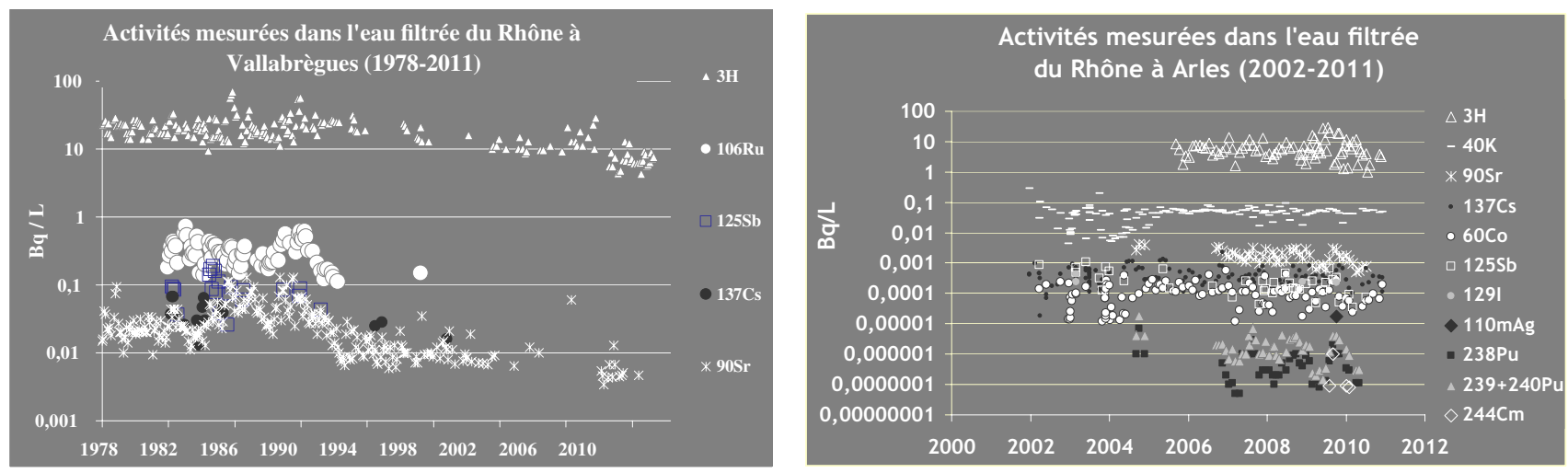

Fig. 2. Chroniques des activités des radionucléides détectés dans les eaux filtrées à Vallabrègues et en Arles (SORA). Time series of radionuclides detected within filtered waters at Vallabrègues and Arles (SORA monitoring station).

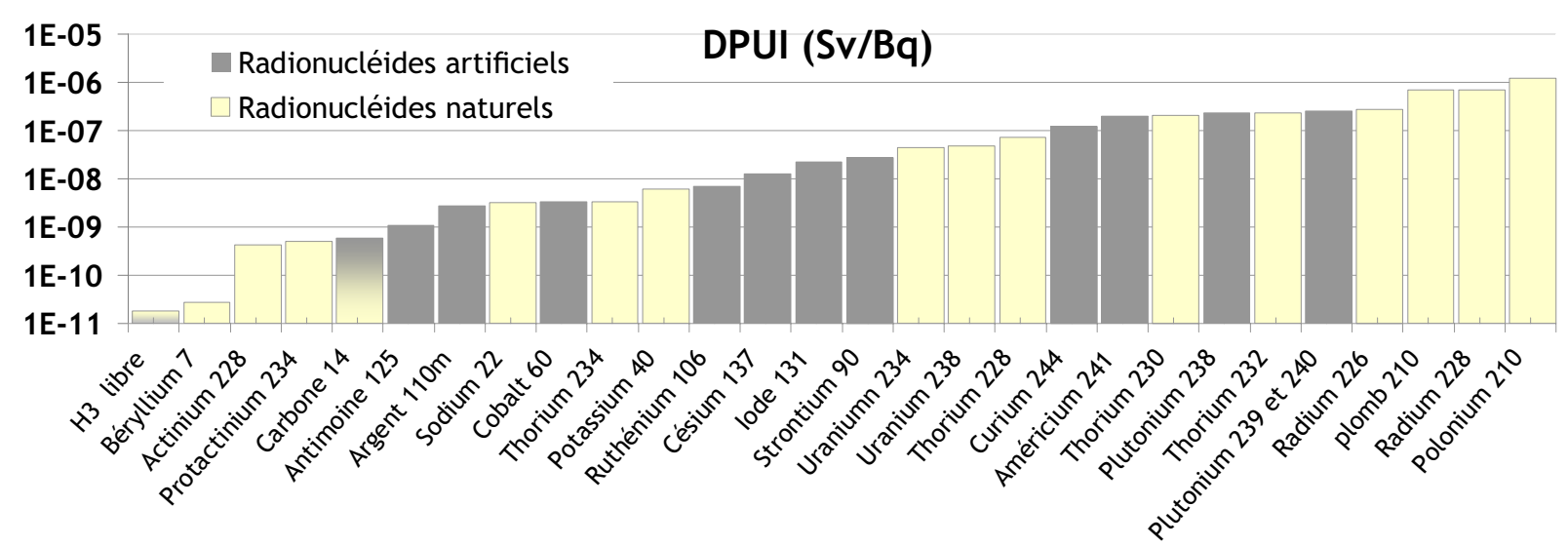

Fig. 3. Facteurs de dose (ou DPUI : Dose Par Unité d'Incorporation) efficace engagée en cas d'ingestion par un adulte (ICRP, 1995); en foncé les radionucléides d'origine artificielle.

Engaged dose factors for adults or DPUI (ICRP, 1995); artificial radionuclides are reported in dark grey.

Le tritium est le radionucléide le plus abondant dans le Rhône. La diminution observée de son activité au cours du temps, de $40 \mathrm{~Bq} / \mathrm{L}$ en moyenne en 1980 à 8 Bq/L actuellement, est liée principalement à la baisse des rejets de Marcoule en 1997. Aujourd'hui, son activité dans le Rhône résulte principalement des rejets des centres nucléaires de production d'électricité (CNPE) (Eyrolle-Boyer et al., 2013). Les activités en tritium du Rhône présentent une gamme de valeurs relativement faibles par rapport à celles observées pour les autres fleuves français sur lesquels sont implantés des CNPE. Ainsi, en 2008, les activités moyennes observées en tritium libre à l'aval de l'ensemble des installations nucléaires étaient comprises entre 6 et $28 \mathrm{~Bq} / \mathrm{L}$ (IRSN, 2009), à comparer aux activités mensuelles mesurées en Arles cette même année, comprises entre 3 et $13 \mathrm{~Bq} / \mathrm{L}$, ainsi qu'à Vallabrègues, comprises entre 6 et $9 \mathrm{~Bq} / \mathrm{L}$. Dans les cours d'eau français hors influence de toute installation nucléaire, les activités en tritium libre sont assez homogènes et inférieures actuellement à $1 \mathrm{~Bq} / \mathrm{L}$ (Eyrolle-Boyer et al., sous presse). Bien que le tritium soit le radionucléide prépondérant des eaux du Rhône il contribue de manière infime à la dose par ingestion (Fig. 3).

Avec une activité moyenne de l'ordre de $0,06 \mathrm{~Bq} / \mathrm{L}$, les isotopes de l'uranium naturel $\left({ }^{238} \mathrm{U}\right.$ et ${ }^{234} \mathrm{U}$ essentiellement) représentent moins de $1 \%$ de l'activité totale de l'eau filtrée, approximativement au même niveau que la somme des activités des ${ }^{226} \mathrm{Ra}$ et ${ }^{228} \mathrm{Ra}$, et le ${ }^{40} \mathrm{~K}$ également d'origine naturelle (Fig. 4). Ces isotopes naturels présentent une radiotoxicité 2000 à 30000 fois plus forte que le tritium (Fig. 3). À des niveaux d'activités moindres, sont détectés dans l'eau filtrée du Rhône au niveau de Vallabrègues depuis le milieu des années soixante-dix, le ${ }^{106} \mathrm{Ru}$, le ${ }^{90} \mathrm{Sr}$, le ${ }^{137} \mathrm{Cs}$ et le ${ }^{125} \mathrm{Sb}$. Depuis 1998 , seul le ${ }^{90} \mathrm{Sr}$, très soluble, provenant d'une part de la rémanence des essais d'armes nucléaires sur le bassin versant du Rhône et d'autre part de la reprise de stocks sédimentaires contaminés par les rejets de Marcoule, est encore détecté dans l'eau filtrée prélevée à Vallabrègues à des niveaux de l'ordre de 0,005 Bq/L (Fig. 2). Les données enregistrées dans l'eau filtrée à Arles entre 2002 et 2011 indiquent des niveaux extrêmement faibles, de l'ordre de 100 à 1000 fois inférieurs à ceux observés dans les années 90 et jusqu'à 5000 fois inférieurs aux activités les plus élevées des années 80 , notamment pour le ${ }^{106} \mathrm{Ru}$. Les activités mesurées pour les isotopes du plutonium ainsi que pour l ${ }^{\prime 241} \mathrm{Am}$ et le ${ }^{244} \mathrm{Cm}$ sont encore 10 à 100 fois plus faibles que les activités précédentes, comprises entre $0,42 \pm 0,25 \mu \mathrm{Bq} / \mathrm{L}$ et $6,3 \pm 1,2 \mu \mathrm{Bq} / \mathrm{L}$ pour $1^{, 241} \mathrm{Am}, 0,72 \pm 0,20$ et $7,0 \pm 0,7 \mu \mathrm{Bq} / \mathrm{L}$ pour ${ }^{239+240} \mathrm{Pu}$ et entre $0,14 \pm 0,13$ et $2,9 \pm 0,4 \mu \mathrm{Bq} / \mathrm{L}$ pour le ${ }^{238} \mathrm{Pu}$ et ${ }^{244} \mathrm{Cm}$. L'ensemble des chroniques montre que, si la radioactivité 


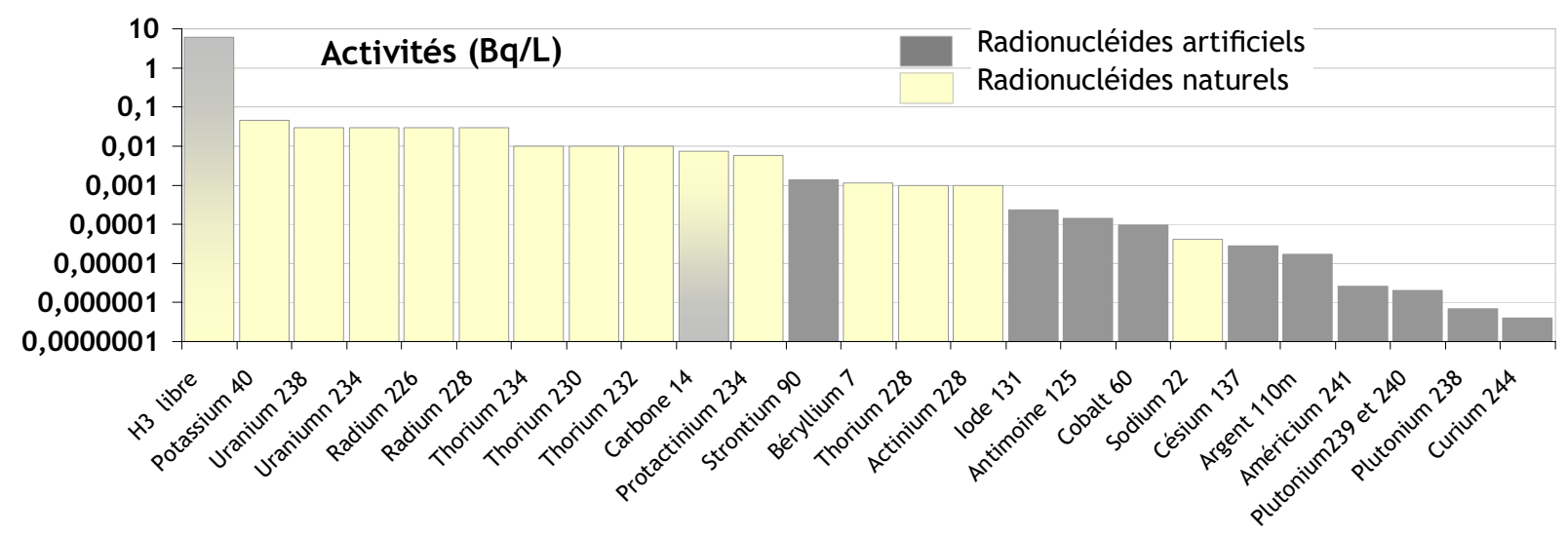

Fig. 4. Activités des principaux radionucléides naturels et artificiels détectés dans les eaux filtrées du Rhône aval en 2011. Activity of natural and artificial radionuclides detected in filtered waters at the lower course of the Rhone River in 2011.

d'origine naturelle demeure bien évidemment constante au cours du temps, comme le soulignent les données enregistrées pour le ${ }^{40} \mathrm{~K}$, les niveaux de contamination des eaux du Rhône ont fortement diminué à partir du début des années 90 , de 5 fois (cas du tritium) à plus de 5000 fois (cas du ${ }^{106} \mathrm{Ru}$ ), en raison de la baisse des rejets liquides de l'installation de Marcoule.

\section{Les résultats d'analyses réalisées en 2011 au sein du canal sur les eaux filtrées}

Le tableau 3 présente les résultats des analyses effectuées sur l'eau prélevée en 2011 au sein du canal Ph. Lamour au niveau de la station de pompage de La Méjanelle. La radioactivité dans l'eau filtrée du canal est essentiellement due au tritium et aux radionucléides d'origine naturelle. Les éléments de filiation des chaînes U-Th mesurés dans les eaux filtrées sont, par ordre d'activité décroissante, ${ }^{210} \mathrm{Po},{ }^{234} \mathrm{U},{ }^{238} \mathrm{U},{ }^{234} \mathrm{~Pa}$, ${ }^{234} \mathrm{Th},{ }^{214} \mathrm{~Pb},{ }^{228} \mathrm{Ac}$ et ${ }^{228} \mathrm{Ra},{ }^{214} \mathrm{Bi},{ }^{210} \mathrm{~Pb}$ et ${ }^{235} \mathrm{U}$. D'une manière générale, les niveaux de radioactivité naturelle mesurés en 2011 dans l'eau filtrée du canal sont dans les gammes des valeurs enregistrées dans les eaux filtrées du Rhône. Parmi les radionucléides artificiels, le ${ }^{3} \mathrm{H}$ présente l'activité volumique la plus élevée $(7,9 \pm 3,4 \mathrm{~Bq} / \mathrm{L})$, supérieure de 4 à 7 ordres de grandeur à l'activité volumique des autres radionucléides artificiels mesurés dans l'eau filtrée. Cette valeur est proche des activités mesurées dans le cadre de la surveillance des eaux du Rhône (Fig. 2). Les autres radionucléides d'origine artificielle détectés dans l'eau filtrée collectée à la Méjanelle sont, le ${ }^{90} \mathrm{Sr}$ $(1500 \mu \mathrm{Bq} / \mathrm{L})$, le ${ }^{137} \mathrm{Cs}(140 \mu \mathrm{Bq} / \mathrm{L}), \mathrm{l}^{1241} \mathrm{Am}$ et les ${ }^{239+240} \mathrm{Pu}$ (de l'ordre de quelques $0,1 \mu \mathrm{Bq} / \mathrm{L}$ ). Ces valeurs sont là encore dans les gammes de valeurs enregistrées par la station SORA.

\subsection{Qualité radiologique des eaux du Rhône aval et dose liée à leur ingestion}

\section{Activité alpha, bêta globale, bêta globale résiduelle et en tritium}

La figure 5 présente l'activité $\alpha$ globale mesurée mensuellement dans l'eau filtrée du Rhône aval de 1978 à 2011. Elle n'a jamais dépassé $0,1 \mathrm{~Bq} / \mathrm{L}$, même dans les années 80 lorsque les actinides émetteurs $\alpha$ rejetés par l'installation de Marcoule (plutonium et américium) influaient légèrement sur la valeur de cet indice. L'activité $\alpha$ globale mesurée dans l'eau du canal aujourd' hui $(0,024 \pm 0,007 \mathrm{~Bq} / \mathrm{L})$ est dans la gamme de valeurs enregistrées actuellement pour les eaux filtrées du Rhône. La figure 6 présente les activités $\beta$ globales, $\beta$ globales résiduelles et du ${ }^{40} \mathrm{~K}$. Jusqu'en 1990, les activités $\beta$ globales étaient très influencées par les rejets de Marcoule $\left({ }^{106} \mathrm{Ru},{ }^{90} \mathrm{Sr}\right.$ et ${ }^{125} \mathrm{Sb}$ notamment) pour une moyenne de $0,3 \mathrm{~Bq} / \mathrm{L}$ sur la période 1978-1990. Avec l'installation de la nouvelle station de traitement des effluents liquides de Marcoule, l'activité $\beta$ globale résiduelle a diminué pour atteindre une moyenne de 0,15 Bq/L sur la période 1991-1998. Avec l'arrêt de l'activité de retraitement du combustible, cette activité $\beta$ globale résiduelle, mesurée désormais dans le Rhône, a encore diminué de moitié (moyenne de $0,07 \mathrm{~Bq} / \mathrm{L}$ depuis 1999). Les activités $\beta$ globales et $\beta$ globales résiduelles mesurées en 2011 dans l'eau filtrée de la Méjanelle sont de 0,161 $\pm 0,048 \mathrm{~Bq} / \mathrm{L}$ et $0,102 \pm 0,048 \mathrm{~Bq} / \mathrm{L}$, respectivement, soit des valeurs non significativement différentes de celles enregistrées aujourd'hui pour les eaux du Rhône à Vallabrègues dans le cadre de la surveillance.

Enfin, l'activité en tritium n'a jamais dépassé $100 \mathrm{~Bq} / \mathrm{L}$ (Fig. 2). Ces dix dernières années, l'activité du tritium observée reste inférieure à $30 \mathrm{~Bq} / \mathrm{L}$ et le plus souvent inférieure à $10 \mathrm{~Bq} / \mathrm{L}$, comme mesuré en 2011 dans l'eau filtrée de la Méjanelle $(7,9 \pm 3,3 \mathrm{~Bq} / \mathrm{L})$.

Aucune des limites d'activités $\alpha$ globale, $\beta$ globale et en tritium n'a donc été dépassée dans l'eau filtrée du Rhône aval au cours des dernières décennies. La dose totale indicative a donc été réputée inférieure à $0,1 \mathrm{mSv} / \mathrm{an}$.

\section{Dose Totale Indicative (DTI)}

Les données disponibles acquises dans le cadre de la surveillance des eaux du Rhône aval permettent une évaluation de la DTI (Fig. 7). Le ${ }^{210}$ Po et les radiums présentent des facteurs de dose plus de 10000 fois plus élevés que celui du ${ }^{3} \mathrm{H}$ (Fig. 3). La règlementation stipule que seuls les radionucléides mesurés sont à prendre en compte; les radionucléides dont les activités sont inférieures aux limites de détection ne sont donc pas à considérer. La surveillance des hydrosytèmes permet 
Tableau 3. Résultats d'analyses de l'eau filtrée collectée au sein du canal BRL à la Méjanelle en 2011*. Inc. : incertitude sur le résultat de mesure. LD : limite de détection. ${ }^{*}: \mathrm{L}^{2}{ }^{236} \mathrm{U}$ naturel est en très faible abondance ; sa détection témoigne généralement d'un apport anthropique. Results for filtered waters collected within the Philippe Lamour channel at the Méjanelle in 2011. Inc.: uncertainty on analysis. LD: detection limit. *: naturally occurring ${ }^{236} \mathrm{U}$ is in very low abundance; its detection generally reflects anthropogenic inputs.

\begin{tabular}{|c|c|c|c|c|c|}
\hline & & \multirow[b]{2}{*}{ Élément } & \multicolumn{2}{|c|}{$\begin{array}{c}\text { Eau filtrée }(\mathbf{0 , 4 5} \boldsymbol{\mu m}) \\
\text { Canal BRL, Station de pompage de la } \\
\text { Méjanelle } \\
\text { prélevé le } 08 / 12 / 2011 \text { Eau filtrée }\end{array}$} & \multirow[b]{2}{*}{ Unité } \\
\hline & & & Valeur & Inc. ou LD & \\
\hline & & Activité alpha globale & 0,024 & 0,007 & $\mathrm{~Bq} / \mathrm{L}$ \\
\hline & & Activité bêta globale & 0,161 & 0,048 & $\mathrm{~Bq} / \mathrm{L}$ \\
\hline & & Activité bêta globale résiduelle & 0,102 & 0,048 & $\mathrm{~Bq} / \mathrm{L}$ \\
\hline & & Potassium & 2,1 & 0,21 & $\mathrm{mg} / \mathrm{L}$ \\
\hline & & Tritium $\left({ }^{3} \mathrm{H}\right)$ & 7,9 & 3,4 & $\mathrm{~Bq} / \mathrm{L}$ \\
\hline & & DTI & 18,2 & & $\mu \mathrm{Sv} / \mathrm{an}$ \\
\hline \multirow{21}{*}{ 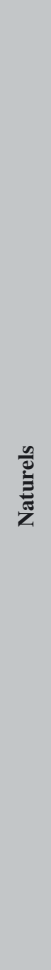 } & $y^{2} \stackrel{0}{0}$ & ${ }^{7} \mathrm{Be}$ & 0,0011 & 0,00022 & $\mathrm{~Bq} / \mathrm{L}$ \\
\hline & 光 & ${ }^{40} \mathrm{~K}$ & 0,0644 & 0,00678 & $\mathrm{~Bq} / \mathrm{L}$ \\
\hline & & ${ }^{228} \mathrm{Ac}$ & 0,0006 & 0,00017 & $\mathrm{~Bq} / \mathrm{L}$ \\
\hline & & ${ }^{212} \mathrm{Bi}$ & $<$ & 0,00271 & $\mathrm{~Bq} / \mathrm{L}$ \\
\hline & ๘ี & ${ }^{212} \mathrm{~Pb}$ & $<$ & 0,00017 & $\mathrm{~Bq} / \mathrm{L}$ \\
\hline & $\stackrel{g}{\Xi}$ & ${ }^{224} \mathrm{Ra}$ & $<$ & 0,00085 & $\mathrm{~Bq} / \mathrm{L}$ \\
\hline & हี & ${ }^{228} \mathrm{Ra}$ & 0,0006 & 0,00017 & $\mathrm{~Bq} / \mathrm{L}$ \\
\hline & & ${ }^{232} \mathrm{Th}$ & $<$ & 0,0007 & $\mathrm{~Bq} / \mathrm{L}$ \\
\hline & & ${ }^{208} \mathrm{Tl}$ & $<$ & 0,00022 & $\mathrm{~Bq} / \mathrm{L}$ \\
\hline & \multirow{10}{*}{ 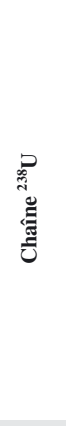 } & ${ }^{214} \mathrm{Bi}$ & 0,0005 & 0,00010 & $\mathrm{~Bq} / \mathrm{L}$ \\
\hline & & ${ }^{234} \mathrm{~Pa}$ & 0,0080 & 0,00322 & $\mathrm{~Bq} / \mathrm{L}$ \\
\hline & & ${ }^{210} \mathrm{~Pb}$ & 0,0005 & 0,00020 & $\mathrm{~Bq} / \mathrm{L}$ \\
\hline & & ${ }^{214} \mathrm{~Pb}$ & 0,0006 & 0,00012 & $\mathrm{~Bq} / \mathrm{L}$ \\
\hline & & ${ }^{210} \mathrm{Po}$ & 0,0153 & 0,00460 & $\mathrm{~Bq} / \mathrm{L}$ \\
\hline & & ${ }^{226} \mathrm{Ra}$ & $<$ & 0,00203 & $\mathrm{~Bq} / \mathrm{L}$ \\
\hline & & ${ }^{230} \mathrm{Th}$ & $<$ & 0,00770 & $\mathrm{~Bq} / \mathrm{L}$ \\
\hline & & ${ }^{234} \mathrm{Th}$ & 0,0069 & 0,00085 & $\mathrm{~Bq} / \mathrm{L}$ \\
\hline & & ${ }^{238} \mathrm{U}$ & 0,0109 & 0,00120 & $\mathrm{~Bq} / \mathrm{L}$ \\
\hline & & ${ }^{234} \mathrm{U}$ & 0,0123 & 0,00200 & $\mathrm{~Bq} / \mathrm{L}$ \\
\hline & $p$ & ${ }^{235} \mathrm{U}$ & 0,000507 & 0,000058 & $\mathrm{~Bq} / \mathrm{L}$ \\
\hline & ฮี & ${ }^{227} \mathrm{Th}$ & $<$ & 0,00120 & $\mathrm{~Bq} / \mathrm{L}$ \\
\hline & \multirow{18}{*}{ 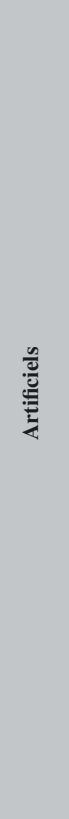 } & ${ }^{110} \mathrm{Ag}$ & $<$ & 0,00029 & $\mathrm{~Bq} / \mathrm{L}$ \\
\hline & & ${ }^{241} \mathrm{Am}$ & $2,0 \times 10^{-7}$ & $1,2 \times 10^{-7}$ & $\mathrm{~Bq} / \mathrm{L}$ \\
\hline & & ${ }^{57} \mathrm{Co}$ & $<$ & 0,00003 & $\mathrm{~Bq} / \mathrm{L}$ \\
\hline & & ${ }^{58} \mathrm{Co}$ & $<$ & 0,00007 & $\mathrm{~Bq} / \mathrm{L}$ \\
\hline & & ${ }^{60} \mathrm{Co}$ & $<$ & 0,00009 & $\mathrm{~Bq} / \mathrm{L}$ \\
\hline & & ${ }^{134} \mathrm{Cs}$ & $<$ & 0,00007 & $\mathrm{~Bq} / \mathrm{L}$ \\
\hline & & ${ }^{137} \mathrm{Cs}$ & 0,00014 & 0,00003 & $\mathrm{~Bq} / \mathrm{L}$ \\
\hline & & ${ }^{54} \mathrm{Mn}$ & $<$ & 0,00007 & $\mathrm{~Bq} / \mathrm{L}$ \\
\hline & & ${ }^{239+240} \mathrm{Pu}$ & $4,7 \times 10^{-7}$ & $3,4 \times 10^{-7}$ & $\mathrm{~Bq} / \mathrm{L}$ \\
\hline & & ${ }^{238} \mathrm{Pu}$ & $<$ & $3,1 \times 10^{-7}$ & $\mathrm{~Bq} / \mathrm{L}$ \\
\hline & & ${ }^{239} \mathrm{Pu}$ & $\mathrm{nm}$ & & $\mathrm{Bq} / \mathrm{L}$ \\
\hline & & ${ }^{240} \mathrm{Pu}$ & $\mathrm{nm}$ & & $\mathrm{Bq} / \mathrm{L}$ \\
\hline & & ${ }^{241} \mathrm{Pu}$ & $\mathrm{nm}$ & & $\mathrm{Bq} / \mathrm{L}$ \\
\hline & & ${ }^{106} \mathrm{Rh}$ & $<$ & 0,00054 & $\mathrm{~Bq} / \mathrm{L}$ \\
\hline & & ${ }^{124} \mathrm{Sb}$ & $<$ & 0,00005 & $\mathrm{~Bq} / \mathrm{L}$ \\
\hline & & ${ }^{125} \mathrm{Sb}$ & $<$ & 0,00015 & $\mathrm{~Bq} / \mathrm{L}$ \\
\hline & & ${ }^{90} \mathrm{Sr}$ & 0,0015 & 0,00030 & $\mathrm{~Bq} / \mathrm{L}$ \\
\hline & & ${ }^{236} \mathrm{U}^{*}$ & $<$ & 0,000008 & $\mathrm{~Bq} / \mathrm{L}$ \\
\hline
\end{tabular}




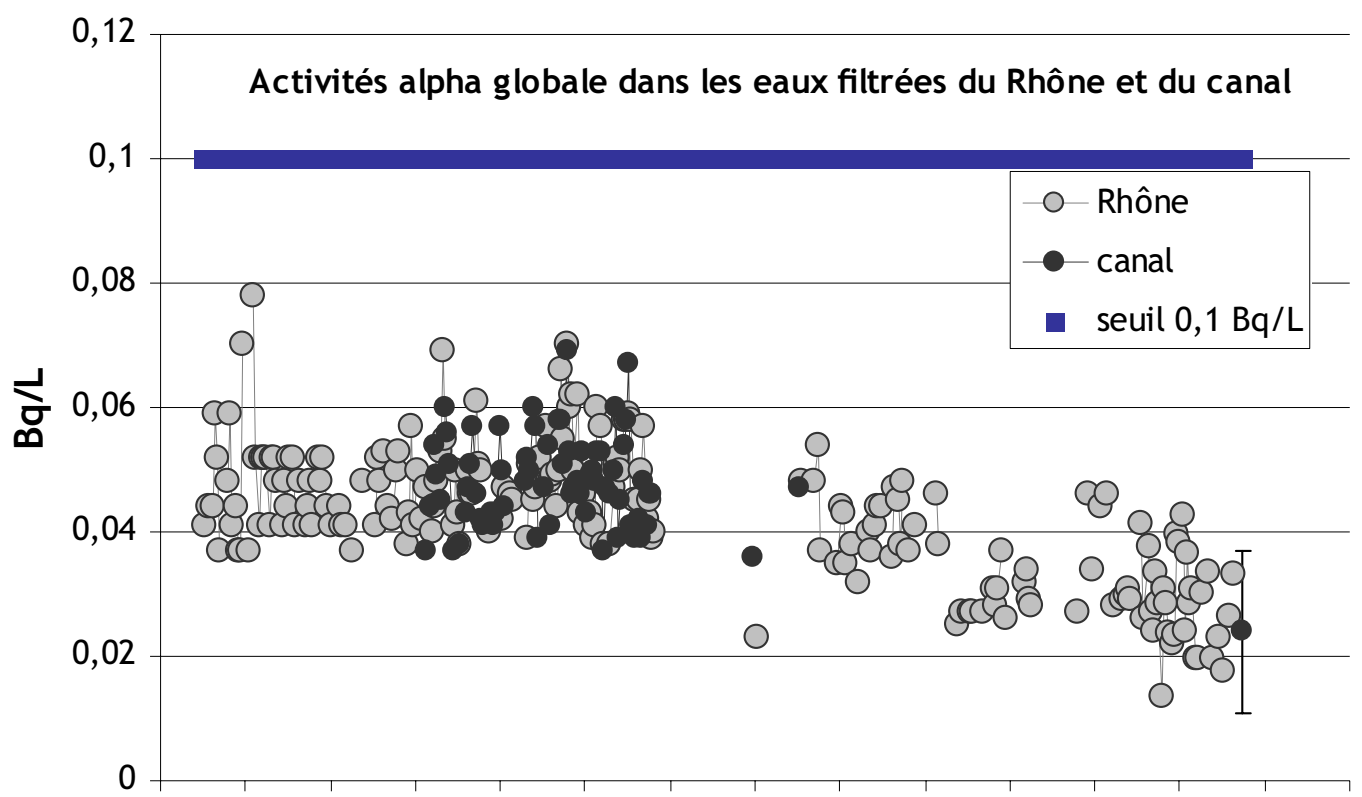

197619791982198419871990199319951998200120042006200920122014

Fig. 5. Évolution de l'activité $\alpha$ globale mesurée dans l'eau filtrée du Rhône à Vallabrègues de 1978 à 2011, du canal BRL à Pichegu de 1985 à 1997 et à la Méjanelle en 2011.

Trend of gross $\alpha$ activity measured in filtered waters at Vallabrègues from 1985 to 1997, within the Philippe Lamour channel at Pichegu and at the Méjanelle in 2011.

de disposer de nombreuses données et chroniques. Ainsi, les estimations faites à partir des données de la surveillance permettent de prendre en considération les principaux radionucléides qui contribuent aux DTI. Les DTI ainsi calculées sont de l'ordre de 3 fois inférieures à la référence. Elles sont principalement imputables à la radioactivité naturelle et notamment aux ${ }^{226} \mathrm{Ra}$ et ${ }^{228} \mathrm{Ra}$. Bien que le tritium représente aujourd'hui plus de $98 \%$ de la radioactivité des eaux filtrées du Rhône, il ne contribue qu'à moins de $1 \%$ de la DTI en raison d'une radio toxicité près de 40000 fois inférieure à celle du ${ }^{228} \mathrm{Ra}$ par exemple. Même en 1982, au moment des rejets parmi les plus importants de Marcoule, la radioactivité artificielle ne représentait que $11 \%$ de la DTI liée à la consommation de l'eau du canal.

En 2011, la DTI déterminée à partir des analyses réalisées sur l'eau filtrée collectée dans le canal $\mathrm{Ph}$. Lamour à la Méjanelle est de 18,2 $\mu \mathrm{Sv} / \mathrm{an}$ (Tab. 3), soit du même ordre de grandeur que les valeurs estimées à partir des données de la surveillance des eaux du Rhône (Fig. 7).

\subsection{Mise en perspective régionale avec les fleuves Orb et Hérault}

Le tableau 4 présente les activités mesurées dans les eaux filtrées de l'Orb et de l'Hérault ainsi qu'un comparatif avec les niveaux observés dans l'eau filtrée du canal. Les seuls radionucléides d'origine artificielle détectés dans l'Orb et l'Hérault sont les ${ }^{3} \mathrm{H},{ }^{90} \mathrm{Sr},{ }^{137} \mathrm{Cs}$ et ${ }^{239+240} \mathrm{Pu}$. Tous ces éléments proviennent des retombées atmosphériques des essais d'armes nucléaires réalisés entre 1945 et 1980 et des retombées de l'accident de Tchernobyl (Renaud et al., 2004). Ces éléments artificiels sont ceux détectés dans les eaux du canal et présentent des niveaux d'activités équivalents, à l'exception du tritium, détecté uniquement dans l'Hérault, qui présente une activité 10 fois plus faible que dans l'eau filtrée du canal. Seul 1 ' ${ }^{241} \mathrm{Am}$, mesuré à un niveau très proche de la limite de détection dans l'eau du canal, n'a pas été décelé dans les eaux filtrées de l'Orb et de l'Hérault. Soulignons que le ${ }^{134} \mathrm{Cs}$ a été quantifié dans l'Hérault à un niveau toutefois très proche de la limite de détection. Cet élément, introduit par les rejets des CNPE mais aussi par les retombées atmosphériques de l'accident de Fukushima en mars 2011 (collectif DEI, 2011), n'était plus détecté dans l'environnement depuis plusieurs années. Concernant la radioactivité naturelle, les niveaux mesurés dans les eaux filtrées des fleuves côtiers étudiés sont soit supérieurs (jusqu'à un facteur 8 pour le ${ }^{210} \mathrm{~Pb}$ ) soit inférieurs (jusqu'à un facteur 5 dans le cas du ${ }^{210} \mathrm{Po}$ ) à ceux de l'eau du canal, témoignant des déséquilibres dans les chaînes U-Th, particulièrement marqués au sein de l'eau filtrée (Picat et al., 2002).

Les rapports d'activité ${ }^{238} \mathrm{U} /{ }^{234} \mathrm{U}$ et ${ }^{238} \mathrm{U} /{ }^{235} \mathrm{U}$ des eaux filtrées des différents échantillons étudiés sont indiqués dans le tableau 5. Certains, en particulier le rapport ${ }^{238} \mathrm{U}^{234} \mathrm{U}$, présentent des écarts significatifs par rapport aux valeurs caractéristiques. Dans les eaux naturelles, les isotopes de l'uranium naturel s'observent en effet aussi bien à l'état d'équilibre qu'en déséquilibre en faveur de l'un ou l'autre des isotopes, d'où l'intérêt de faire des mesures isotopiques pour calculer la dose indicative (Loyen et Thomassin, 2009; Loyen et Brassac, 2012).

Les DTI déterminées dans les eaux filtrées de l'Hérault et de l'Orb en 2011 sont de 11,6 $\mu \mathrm{Sv} / \mathrm{an}$ et 3,5 $\mu \mathrm{Sv} / \mathrm{an}$ en prenant en considération les éléments pour lesquels l'activité à été quantifiée (Fig. 8). Les valeurs des DTI dépendent fortement des éléments quantifiés, et par conséquent ne peuvent pas être 

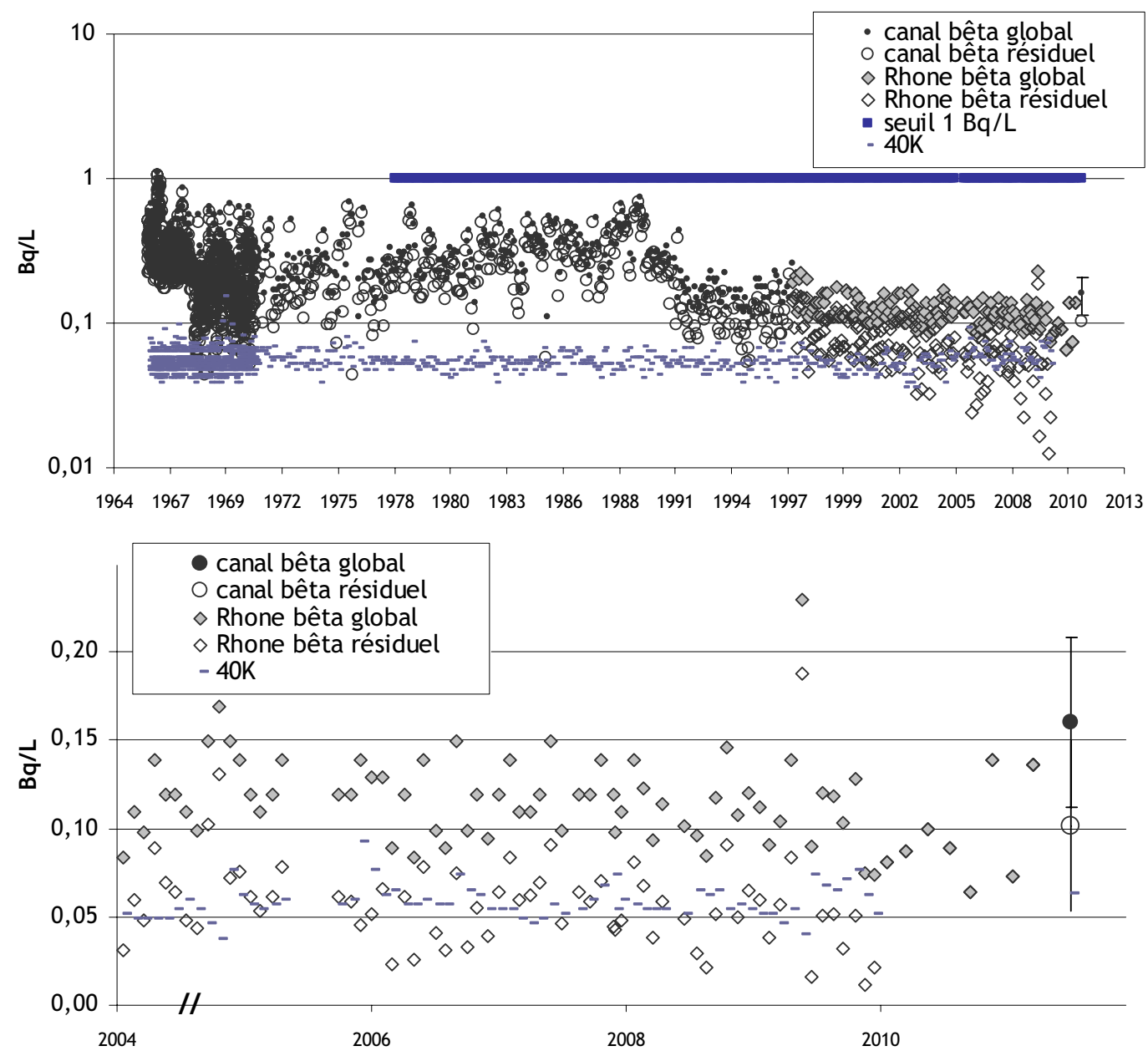

Fig. 6. Activités $\beta$ globales, $\beta$ globales résiduelles (activités du ${ }^{40} \mathrm{~K}$ déduites) et du ${ }^{40} \mathrm{~K}$ dans les eaux filtrées du canal à Pichegu jusqu'en mai 1997, à la Méjanelle en 2011 et dans celles du Rhône après mai 1997.

Gross $\beta$ activity, residual $\beta$ activity (without ${ }^{40} \mathrm{~K}$ contribution) and ${ }^{40} \mathrm{~K}$ activity measured in filtered waters within the Philippe Lamour channel at Pichegu until May 1997 and at the Méjanelle in 2011 and in the downstream Rhone River after May 1997.

\section{DTI en $2011: 28 \mu \mathrm{Sv} / \mathrm{an}$}

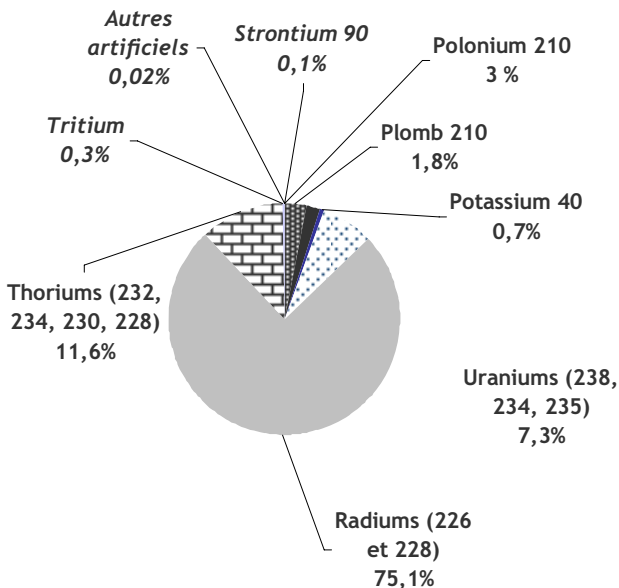

DTI en 1982: $31 \mu \mathrm{Sv} / \mathrm{an}$

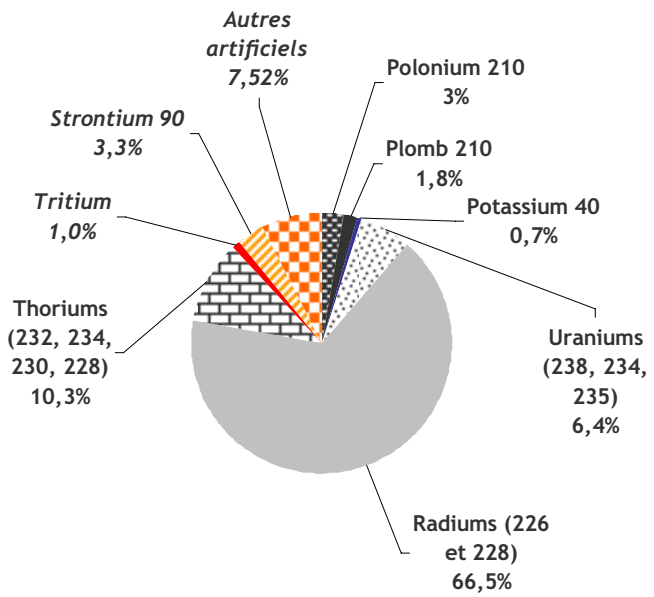

Fig. 7. Doses totales indicatives liées à l'ingestion d'eau filtrée du Rhône en 1982 et en 2011, et principaux radionucléides contributeurs. Total Indicative Dose due to consumption of filtered water from the downstream Rhone River in 1982 and then in 2011. Main radionuclides contributing to the dose. 
Tableau 4. Résultats d'analyses des eaux filtrées collectées dans les fleuves côtiers Orb et Hérault et comparaison aux eaux filtrées du canal prélevées à la Méjanelle, *. Inc. : Incertitude sur le résultat de mesure. LD : Limite de détection. L ${ }^{236} \mathrm{U}$ naturel est en très faible abondance ; sa détection témoigne généralement d'un apport anthropique.

Results for filtered waters collected within the Orb and Hérault rivers and their comparison with filtered waters collected within the Philippe Lamour channel at the Méjanelle. Inc. : uncertainty on analysis. LD : Detection Limit. * : naturally occurring ${ }^{236} \mathrm{U}$ is in very low abundance ; its detection generally reflects anthropogenic inputs.

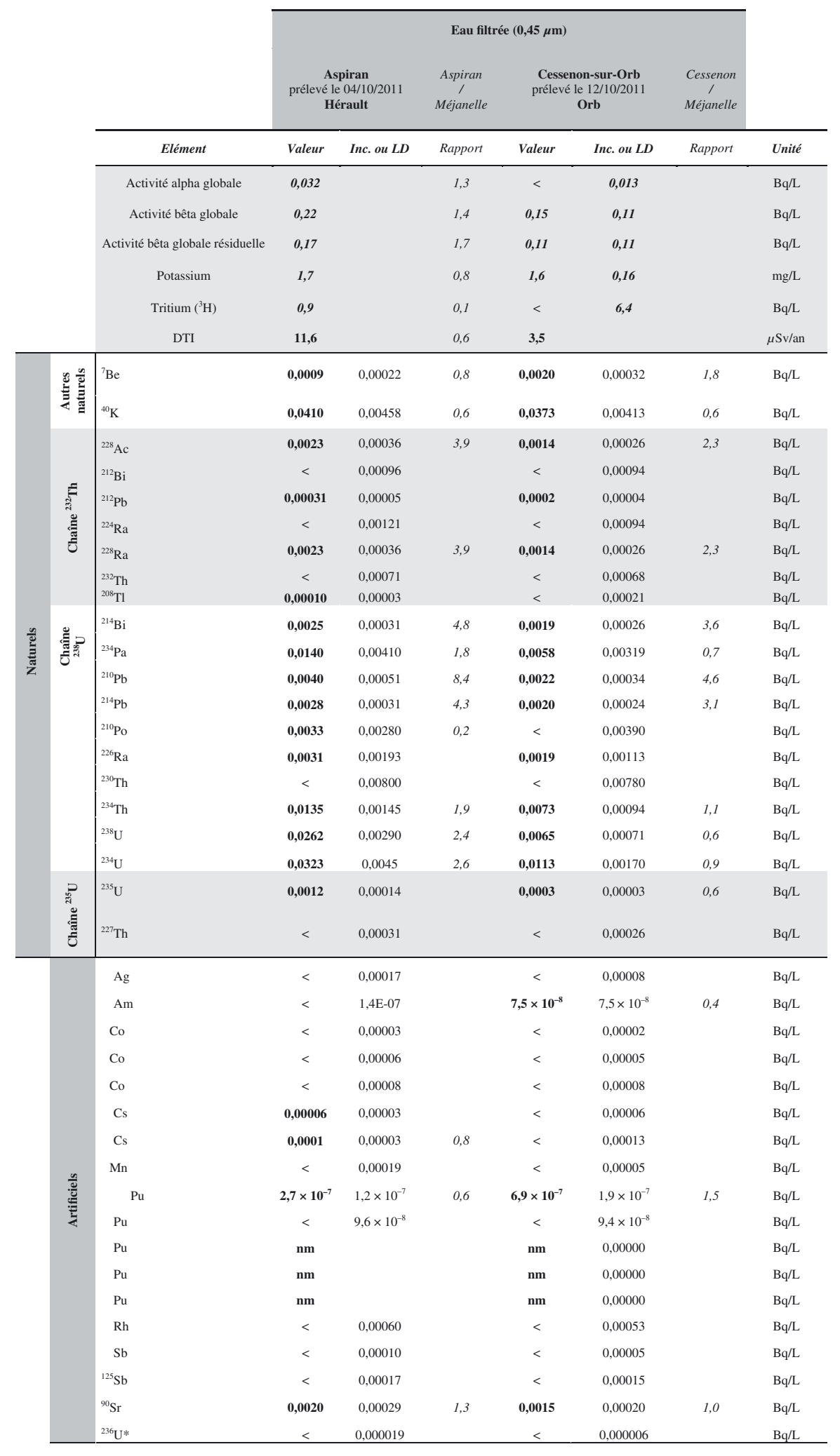




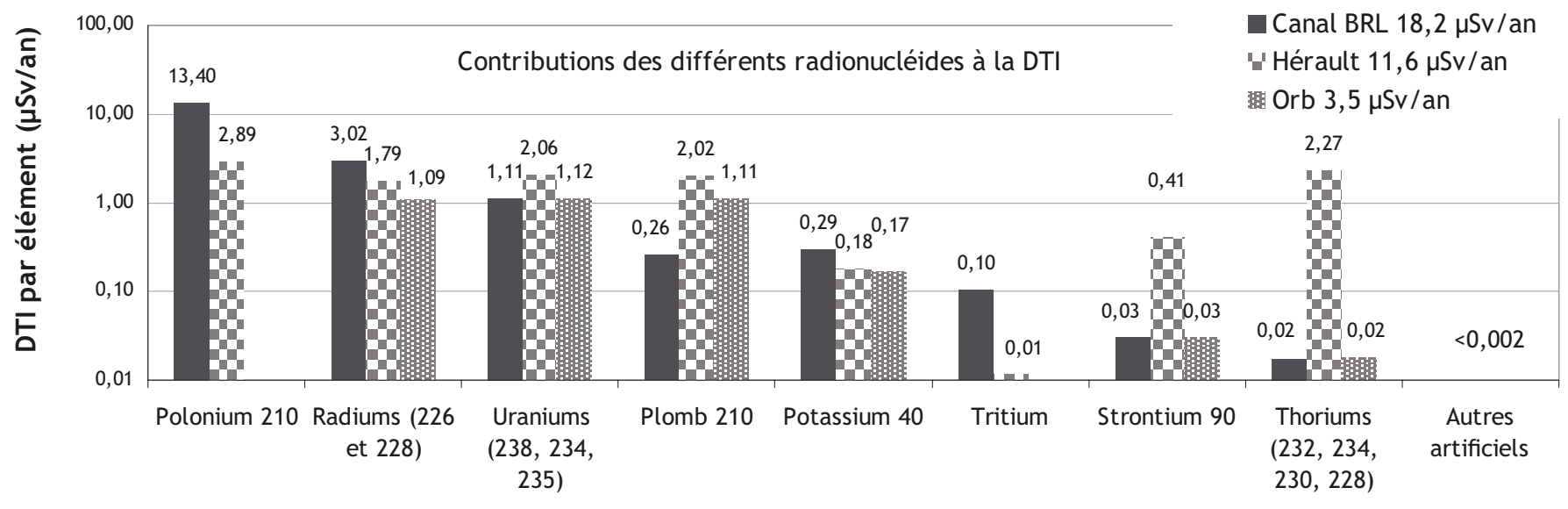

Fig. 8. Contribution des différents radionucléides à la DTI lorsqu'ils ont été mesurés en 2011 dans l'eau filtrée du canal, de l'Hérault et de l'Orb.

Contribution of the various radionuclides to the Total Indicative Dose when measured in 2011 in the filtered waters of the Philippe Lamour Channel and the Orb and Hérault coastal rivers.

Tableau 5. Rapports d'activité ${ }^{238} \mathrm{U} /{ }^{234} \mathrm{U}$ et ${ }^{238} \mathrm{U} /{ }^{235} \mathrm{U}$ au sein des eaux filtrées des différents échantillons étudiés et comparaison aux valeurs caractérisant l'uranium naturel.

${ }^{238} \mathrm{U} /{ }^{234} \mathrm{U}$ and ${ }^{238} \mathrm{U} /{ }^{235} \mathrm{U}$ activity ratios for filtered waters studied and comparison with natural uranium.

\begin{tabular}{ccccc} 
& \multicolumn{4}{c}{ Eau filtrée } \\
& $\mathbf{U}_{\text {Naturel }}$ & Canal & Hérault & Orb \\
\hline${ }^{238} \mathbf{U} /{ }^{234} \mathbf{U}$ & 1 & 0,9 & 0,8 & 0,6 \\
${ }^{238} \mathbf{U} /{ }^{235} \mathbf{U}$ & 21 & 21,5 & 22,0 & 21,7 \\
\hline
\end{tabular}

directement comparées les unes aux autres. Dans tous les cas, il est important de souligner que les DTI sont très inférieures à la valeur de référence de $0,1 \mathrm{mSv} / \mathrm{an}$, que ce soit dans le canal, l'Orb ou l'Hérault. La contribution à la DTI des radionucléides artificiels détectés dans ces hydrosystèmes est en outre négligeable $(<0,01 \%)$.

\section{Conclusions}

Les données acquises en 2011 sur les eaux filtrées du canal Philippe Lamour du Réseau hydraulique régional propriété de la région et géré par BRL, et celles enregistrées par les stations de surveillance des eaux filtrées du Rhône sur sa partie aval ont permis d'évaluer la qualité radiologique des eaux destinées à la consommation depuis les années 1980 jusqu'à nos jours. Cette évaluation a été faite, conformément à la règlementation relative aux eaux destinées à la consommation humaine, à partir de quatre indicateurs : l'activité $\alpha$ globale, l'activité $\beta$ globale résiduelle, l'activité du tritium et la dose totale indicative (DTI).

Nos résultats montrent que les activités $\alpha$ globale et $\beta$ globale résiduelle mesurées dans les eaux filtrées du Rhône aval se situent actuellement respectivement plus de 2 fois et plus de 10 fois en dessous des seuils d'investigation, celles du tritium 10 à 30 fois en dessous. La dose totale indicative calculée sur la base des radionucléides recherchés et mesurés a été estimée comme étant 3 fois inférieure à la valeur de référence de $0,1 \mathrm{mSv} / \mathrm{an}$, plus de $99 \%$ de cette dose étant dus à la radioactivité naturelle. Ces seuils d'investigation, fixés par la règlementation sont eux-mêmes très en-deçà de niveaux de potabilité, notamment pour le tritium pour lequel la limite de potabilité recommandée par l'OMS est de $10000 \mathrm{~Bq} / \mathrm{L}$, soit de l'ordre de 1000 fois supérieure à l'activité moyenne observée dans les eaux du Rhône aval et dans le canal. L'exploitation des données issues des chroniques de la surveillance montre en outre qu' aucune des limites d'activités $\alpha$ globale, $\beta$ globale résiduelle et en tritium, définies par l'article R.1321-20 du code de la santé publique, n'a donc été dépassée dans l'eau filtrée du Rhône aval au cours des dernières décennies. La dose totale indicative des eaux filtrées du Rhône aval a donc été inférieure à $0,1 \mathrm{mSv} / \mathrm{an}$. Les DTI déterminées dans les eaux filtrées de l'Hérault et de l'Orb en 2011 ne sont pas fondamentalement différentes de celles déterminées pour les eaux du Rhône aval. Elles dépendent à plus de 99,99\% des radionucléides d'origine naturelle présents et quantifiés, en particulier le ${ }^{210} \mathrm{Po}$ et les isotopes du radium (226 et 228), du thorium (228, 230, 232 et 234 ) et de l'uranium $(234,235$ et 238$)$.

Remerciements. Les auteurs remercient l'Agence de l'Eau Rhône Méditerranée Corse pour le soutien financier de la Station SORA ainsi que l'OSR (Observatoire des Sédiments du Rhône) de la ZABR (Zone Atelier Bassin du Rhône).

\section{Références}

Antonelli C., Eyrolle F., Boullier V., Gurriaran R., Cossonnet C. (2012) Mesures, estimation et bilan des flux de radioactivité exportés par le Rhône en Méditerranée. Dans : I.S.Rivers, $1^{\text {ère }}$ Conférence Internationale, 26-28 juin 2012, Lyon, France.

Code de la santé publique CSP (Partie réglementaire) (2007) Première partie; Protection générale de la santé - Livre 3; Protection de la santé et environnement - Titre 3; Prévention des risques sanitaires liés à l'environnement et au travail - Chapitre 3; Rayonnements ionisants - Articles R1333-8.

Collectif DEI (2011) Analyse de l'impact de l'accident de Fukushima en France (métropole et DROM-COM) à partir des résultats de la surveillance renforcée de la radioactivité de l'environnement, Rapport IRSN/DEI/2011-01, 90 p. 
Eyrolle F., Renaud Ph. (2012) Qualité radiologique des eaux du canal Bas-Rhône-Languedoc, Synthèse de connaissances, Mise en perspective historique et régionale, rapport final, Rapport IRSN PRP-ENV/SESURE/2012-07.

Eyrolle F., Radakovitch O., Raimbault P., Charmasson C., Antonelli C., Ferrand E., Raccasi G., Aubert D., Gurriaran R. (2012) Consequences of hydrological events on suspended sediment and associated radionuclide deliveries from the Rhône River towards the Mediterranean Sea, Journal of Soils and Sediments 12, 1479-1495.

Eyrolle-Boyer F., Claval D., Antonelli C., Tournieux D., Cossonnet C. (2013) 1963-2013: Fifty years of anthropogenic tritium in our environment - Focus on the Rhône valley (South east France). In: 10th International Conference on Tritium Science and Technology, "TRITIUM 2013”, October 21-25, 2013, Nice Acropolis, France.

Eyrolle-Boyer F., Claval D., Charmasson S., Boyer P., Cossonnet C., Apparent enrichment of organically bound tritium in rivers explained by the heritage of our past, J. Environ. Radioact., sous presse.

ICRP Publication 72 (1995) Age-dependent Doses to the Members of the Public from Intake of Radionuclides - Part 5 Compilation of Ingestion and Inhalation Coefficients, Ann. ICRP 26 (1).
IRSN (2006) Exposition de la population française aux rayonnements ionisants.

IRSN (2009) Le tritium dans l'environnement, Rapport IRSN/DEI 2009-5.

Loyen J., Thomassin A. (2009) Eaux destinées à la consommation humaine présentant une activité alpha globale $>0,1 \mathrm{~Bq} \cdot \mathrm{l}^{-1}$, Bilan 2004-2008, Analyse de la radioactivité - Doses par ingestion, Rapport IRSN DEI 2009-02.

Loyen J., Brassac A. (2012) Profil radiologique des eaux d'adduction présentant une DTI supérieure à $0,1 \mathrm{mSv} / \mathrm{an}$, Exploitation des résultats d'analyses de la radioactivité obtenus entre 2004 et 2011 à l'IRSN, Rapport PRP-ENV/STEME 2012-04.

Picat Ph., Calmet D., Louvat D., Vray F., Lemaitre N., Linden G., Barbey P., Pigree G., Bourcier T., Levy F., Le Bar S., Baron Y., Delacroix D., Panaïva E., De Bruyne T., Hervé J.Y., Beguinel P., Cabanne N., De Paepe A., Santucci C., Prot T., Matray J.L., Tillie J.L. (2002) Radioactivité d'origine naturelle dans l'environnement en France : niveaux non pertubés par l'homme, Radioprotection 37 (3), 283-327.

Renaud Ph., Métivier J.M., Castellier J.M., Pourcelot L., Louvat D. (2004) Cartographie des dépôts de ${ }^{137} \mathrm{Cs}$ en mai 1986 sur l'ensemble du territoire français métropolitain, Radioprotection 39 (1), 23-38.

Cite this article as: F. Eyrolle-Boyer, Ph. Renaud, D. Claval, D. Tournieux, F. Le Dore, J.-F. Blanchet, J. Loyen, Ch. Antonelli, C. Cossonnet, X. Cagnat. Qualité radiologique des eaux filtrées du Rhône aval en vue de la production d'eau destinée à la consommation humaine. Radioprotection 49(3), 183-193 (2014). 